\title{
ANALYZING RCD30 OBLIQUE PERFORMANCE IN A PRODUCTION ENVIRONMENT
}

\author{
M. E. Soler ${ }^{\text {a }}$ W. Kornus, A. Magariños, M.Pla
}

\begin{abstract}
Institut Cartogràfic i Geològic de Catalunya, 08038 Barcelona, Spain - (eulalia.soler, wolfgang.kornus, antonio.magarinos, maria.pla)@icgc.cat
\end{abstract}

\section{Commission III, WG III/1}

KEY WORDS: Oblique imagery, Point Cloud, Mesh, Accuracy Analysis, Normalized Difference Vegetation Index, Line Spread Function.

\begin{abstract}
:
In 2014 the Institut Cartogràfic i Geològic de Catalunya (ICGC) decided to incorporate digital oblique imagery in its portfolio in response to the growing demand for this product. The reason can be attributed to its useful applications in a wide variety of fields and, most recently, to an increasing interest in $3 \mathrm{~d}$ modeling. The selection phase for a digital oblique camera led to the purchase of the Leica RCD30 Oblique system, an 80MPixel multispectral medium-format camera which consists of one Nadir camera and four oblique viewing cameras acquiring images at an off-Nadir angle of $35^{\circ}$. The system also has a multi-directional motion compensation on-board system to deliver the highest image quality.
\end{abstract}

The emergence of airborne oblique cameras has run in parallel to the inclusion of computer vision algorithms into the traditional photogrammetric workflows. Such algorithms rely on having multiple views of the same area of interest and take advantage of the image redundancy for automatic feature extraction. The multiview capability is highly fostered by the use of oblique systems which capture simultaneously different points of view for each camera shot. Different companies and NMAs have started pilot projects to assess the capabilities of the 3D mesh that can be obtained using correlation techniques. Beyond a software prototyping phase, and taking into account the currently immature state of several components of the oblique imagery workflow, the ICGC has focused on deploying a real production environment with special interest on matching the performance and quality of the existing production lines based on classical Nadir images.

This paper introduces different test scenarios and layouts to analyze the impact of different variables on the geometric and radiometric performance. Different variables such as flight altitude, side and forward overlap and ground control point measurements and location have been considered for the evaluation of aerial triangulation and stereo plotting. Furthermore, two different flight configurations have been designed to measure the quality of the absolute radiometric calibration and the resolving power of the system.

To quantify the effective resolution power of RCD30 Oblique images, a tool based on the computation of the Line Spread Function has been developed. The tool processes a region of interest that contains a single contour in order to extract a numerical measure of edge smoothness for a same flight session. The ICGC is highly devoted to derive information from satellite and airborne multispectral remote sensing imagery. A seamless Normalized Difference Vegetation Index (NDVI) retrieved from Digital Metric Camera (DMC) reflectance imagery is one of the products of ICGC's portfolio. As an evolution of this well-defined product, this paper presents an evaluation of the absolute radiometric calibration of the RCD30 Oblique sensor. To assess the quality of the measure, the ICGC has developed a procedure based on simultaneous acquisition of RCD30 Oblique imagery and radiometric calibrated AISA (Airborne Hyperspectral Imaging System) imagery.

\section{INTRODUCTION}

The Institut Cartogràfic i Geològic de Catalunya (ICGC) is the mapping agency of the Government of Catalonia. Created in late 1982, ICGC's activities have been focused on providing valuable geoinformation for territorial planning by generating raster and vector databases at different scales ranging from 1:1 000 to 1:1 000000 . Nowadays, although maintaining up-to-date information at smaller scales, the ICGC is also very concerned about the specific geoinformation required for managing the ever growing urban areas (UN World Urbanization Trend forecasts that $86 \%$ of the population of the developed world will live in urban areas by 2050). This effort is not only a matter of working at large scales (1:1 000 and larger), but also to adopt and develop the specific techniques which better describe the urban ecosystem from many different points of view: urban morphology, energy efficiency, sustainability and environmental applications, etc.

Some of the environmental or energy efficiency applications developed at ICGC for urban planning (such as light pollution, thermal efficiency, Urban Heat Island analysis) are related to specific hyperspectral surveys, but there is an increasing requirement of including detailed and photorealistic 3D models for specific morphological analysis and as a base layer of the urban information infrastructure, moving from $2 \mathrm{D}$ to $3 \mathrm{D}$ environments. 

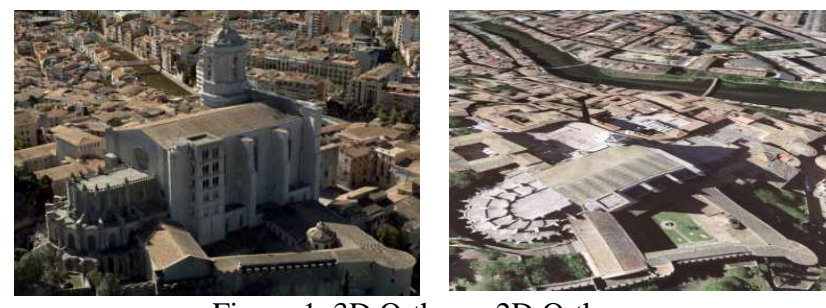

Figure 1.3D Ortho vs 2D Ortho.

LoD1 city models are being regularly produced in urban areas at ICGC as a by-product of the 1:1 000 topographic database. LoD2 city models from LiDAR point clouds are also generated as intermediate information when developing specific energy efficiency applications such as photovoltaic suitability.

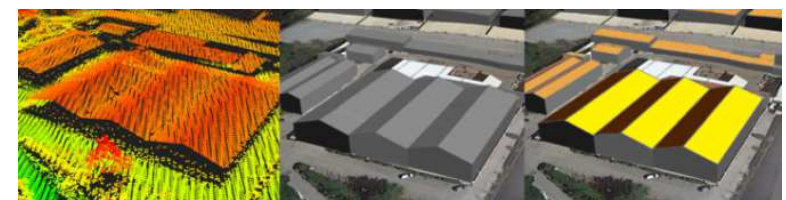

Figure 2. Point cloud. LoD2 buildings. LoD2 building + photovoltaic suitability

The ICGC acquired the oblique camera system in 2014 with the objective of adding a new urban sensor to its portfolio, which enables not only the creation of photorealistic 3D meshes using multi-view pixel-based algorithms, but also the evolution of the current product portfolio. In such evolution, ICGC's analysis aims at increasing the productivity of the oblique flight surveys by maximizing the number of applications derived from the captured information, including 3D meshes, point cloud and façade textures, NDVI orthoimagery and stereo-models for data capture at 1:1000 scale.

Establishing robust production pipelines relying on the oblique camera system requires an in-depth study of the sensor's capabilities. Therefore, this paper presents the current ICGC's analysis of the oblique system based on medium-format RCD30 cameras. The analysis focuses on different aspects of the system. On the one hand, the study of geometric and radiometric suitability of the RDC30 camera for remote sensing applications and stereo plotting at urban scales; on the other hand, the study of the automated aerial triangulation of Nadir and oblique blocks which is challenged by the number of images involved in the high-overlap flight surveys, the scale and resolution variations along the oblique images and the performance of dense point matching across the different viewing directions.
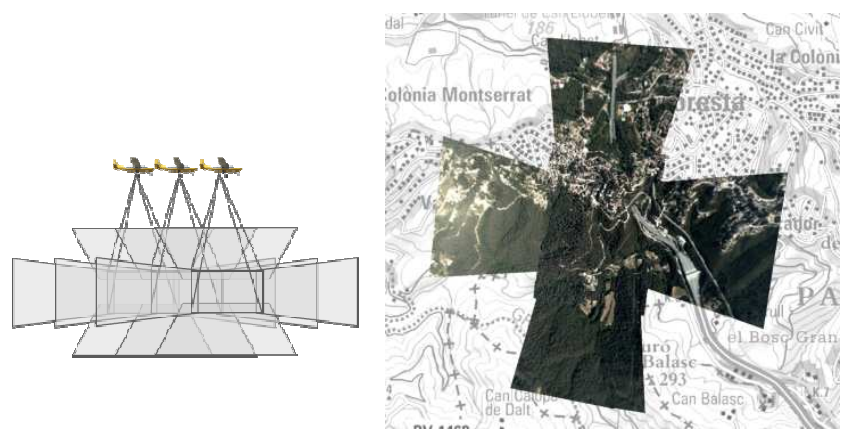

Figure 3. High overlap data capture in maltese-cross configuration

\section{RCD30 OBLIQUE TEST FLIGHTS}

From the whole set of models present in the market, the ICGC finally decided to acquire in 2014, the RCD30 oblique featured by Leica: a $60 \mathrm{Mpixel}$ multispectral medium format camera which consists of one Nadir and four oblique cameras viewing towards the four cardinal points at an off-Nadir angle of $35^{\circ}$. The system can easily be upgraded to 80 Mpixels and has a multi-directional motion compensation on-board system to deliver high quality images.

Three different block configurations have been considered to carry out the camera assessment:

T1: Geometric study has been performed with a block of 10095 images collected with the RCD30 Oblique camera from $770 \mathrm{~m}$ flying altitude (AGL) with $80 \%$ sidelap and $70 \%$ endlap. The block was flown in northeast - south-west direction, 36 strips and a resulting GSD of $8 \mathrm{~cm}$. About 45 shots per flight line were taken from each camera.

T2: For the radiometric assessment, a simultaneous flight with the AISA Eagle II hyperspectral sensor was planned. The configuration chosen, with a flying height of $1650 \mathrm{~m}$ and 120 knot of speed, produced 126-band AISA four strips images with a resulting GSD of $1 \mathrm{~m}$. At the same time, a block of 240 images was collected with the RCD30 Oblique with $60 \%$ sidelap, $40 \%$ endlap and $17 \mathrm{~cm}$ GSD.

T3: A block of 240 images collected from $730 \mathrm{~m}$ flying altitude with $60 \%$ sidelap and enlap was acquired for spatial resolution evaluation. The resolution pattern was located in different parts of the image. The resulting GSD for this configuration was 7.5 $\mathrm{cm}$.

For the acquisition process, the RCD30 Oblique was mounted on a PAV gyro-stabilized suspension mount. The weather conditions during the flight tests were excellent.

\section{AERIAL TRIANGULATION}

A sub-block of 866 images extracted from the T1 configuration has been triangulated using the Trimble Inpho v7.0.2 software package (Match-AT). Approximately 11 images per flight line were taken from each camera. For accuracy assessment a total of 16 control and check points were available, evenly distributed over the block.

The simultaneous image capture with 5 cameras and the high image overlap resulted in a very high degree of image connections causing long processing times for image matching. Although the setting parameters of the matching module were highly restrictive to reduce the amount of matched points as much as possible, an average of 700 points per image were correlated including points connecting up to 112 images. The process took 31 hours on a 64-bit server (16GB RAM, 16 threads, local image storage). After thinning out the dense point distribution to approximately 100 points per image, the block adjustment took less than 10 minutes.

The following input data was introduced into the adjustment: approximately 87000 image point coordinates with an a priori standard deviation $\sigma$ of 2 microns (automatic points) and 3 microns (manual points), GNSS coordinates of the projection centres $(\sigma=3 \mathrm{~cm}$ for $\mathrm{X}, \mathrm{Y}$ and $\mathrm{Z})$, IMU angle observations (Nadir looking camera only; $\sigma=3 \mathrm{mdeg}$ for omega and phi, $\sigma=15$ mdeg for kappa) and ground coordinates of 4 control 
points $(\sigma=4 \mathrm{~cm}$ for $\mathrm{X}, \mathrm{Y}$ and $\mathrm{Z})$. The adjustment settings considered shift and drift parameters of the GNSS measurements, IMU misalignment and self-calibration (44 parameters for each camera).

Self-calibration led to significantly improved results. The Trimble Inpho software allows applying two different sets of self-calibration parameters: the 12 so-called Ebner parameters (Ebner, 1976) and the 44 so-called Grün parameters (Grün, 1979). Figure 4 shows the averaged residuals of image coordinates for the Nadir image derived from block adjustment without self-calibration. The mayor systematic effects could be compensated by applying self-calibration with 44 parameters. The set of 12 parameters did not result in satisfying object point accuracy.

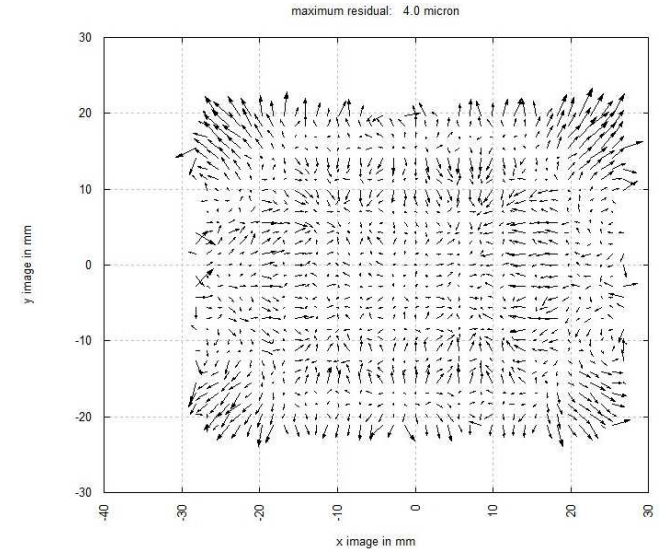

Figure 4. Calculated distortion grid for Nadir camera

From 12 independent check points, RMS object point residuals of $3.2 \mathrm{~cm}$ in $\mathrm{X}, 4.5 \mathrm{~cm}$ in $\mathrm{Y}$ and $6.6 \mathrm{~cm}$ in $\mathrm{Z}$ were obtained. Figure 5 shows the graphical representation of the ground control and check point residuals.
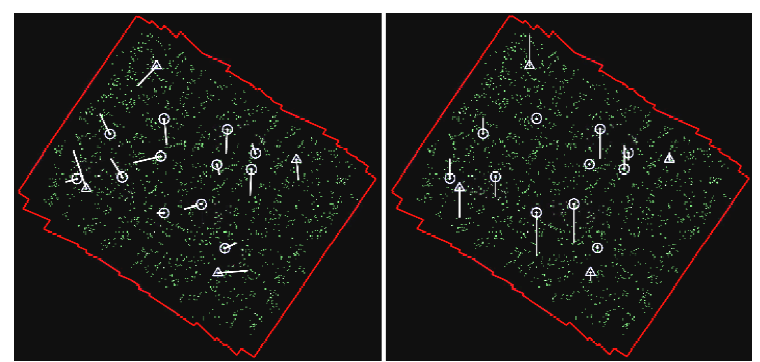

Figure 5. Horizontal (left) and vertical (right) residuals of ground control points (triangles) and check points (circles)

This well characterized block-configuration has been the one preferred to generate preliminary dense point clouds and subsequent textured meshes using the Bentley Smart3DContextCapture software package (Acute3d).

\section{RADIOMETRIC CALIBRATION}

The availability of remote sensing data, either from satellites or manned and unmanned airborne platforms, is growing more and more due to their useful applications in a wide variety of fields. Airborne photogrammetric systems are able to provide highresolution imagery over large areas in a timely manner, being increasingly used for different applications such as land-use monitoring and environmental conditions assessment. As a consequence, as well as geometric precision, radiometric integrity becomes a key task of data processing for the exploitation of photogrammetric data. The ICGC has been increasingly emphasizing the role of absolute radiometric calibration through the incorporation of innovative products involving radiometric calibration of data into its portfolio. This step basically translates the recorded digital numbers (DNs) into physical information (radiance) using correction coefficients at pixel level that are estimated by integrating sphere in-lab measurements. As a matter of fact, even photogrammetric sensor manufacturers, usually devoted to the design of geometrically high-performance systems, take great care of the absolute calibration of their sensors. Nowadays, they are able to provide the constant values to customers and, at best, to include a workflow to generate radiometrically-corrected images automatically.

The aim of this section is to assess the quality of the absolute calibration workflow included in the image post-processing software package for the RCD30 Penta Oblique camera model operated at ICGC. To evaluate the quality of these measures, ICGC has developed a procedure based on a simultaneous acquisition of RCD30 oblique imagery and the AISA Eagle II hyperspectral sensor.

The AISA Eagle II, which is regularly operated by ICGC, is calibrated once a year by SPECIM. Once it returns to our facilities after calibration and maintenance operations, a systematic validation of its radiometric precision is carried out. This protocol follows these steps:

1. AISA Eagle II sensor is placed about $20 \mathrm{~cm}$ far from the Integrating Sphere

2. Acquisitions of (approx.) 500 lines and 5 milliseconds of dark lines are carried out.

3. Image is radiometrically-corrected using pixel-bypixel calibration coefficients.

4. Radiance information of illuminated pixels is timeaveraged (being the image taken at a distance from the sphere, only the central part of the field of view is illuminated).

5. Radiance is compared with the spectral radiation curve of the Integrating Sphere provided by NPL (National Physical Laboratory) in UK. Figure 6 shows the resulting comparison.

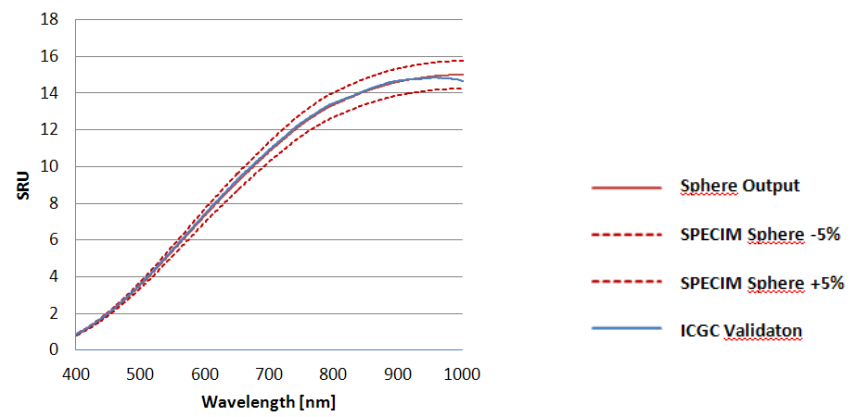

Figure 6. Absolute radiometric calibration of AISA Eagle II validation at ICGC 
The validation procedure demonstrates the reliability of the AISA absolute calibration as a reference to assess the radiometric performance of RCD30 images. With this aim, the 126 useful AISA spectral bands (T2 block-configuration) have been integrated according to RCD30 spectral filters leading to 4-bands imagery that can be used for the assessment in a consistent manner.

The evaluation has been carried out for each channel separately over seven terrain samples representing a heterogeneous set of land covers (e.g. forest, field, asphalt, bare soil, etc.). Thus, a difference image has been calculated from both AISA and RCD30 acquisitions. The statistics computed for each sample show an averaged difference of $22.91 \mathrm{~W} / \mathrm{m}^{2} \mathrm{sr}^{-1} \mu \mathrm{m}$ between blue band calibrated images. Moreover, the averaged difference values found in green, red and near infrared channels are 36.33, 59.66 and $37.04 \mathrm{~W} / \mathrm{m}^{2} \mathrm{sr}^{-1} \mu \mathrm{m}$ respectively. Figures 7, 8, 9 and 10 show the mean values obtained from the histogram of each sample in both AISA and RCD30 calibrated images.
Calibrated Digital Numbers

$\left[\mathrm{Wm}^{-2} \mathrm{sr}^{-1} \mu \mathrm{m}^{-2}\right]$

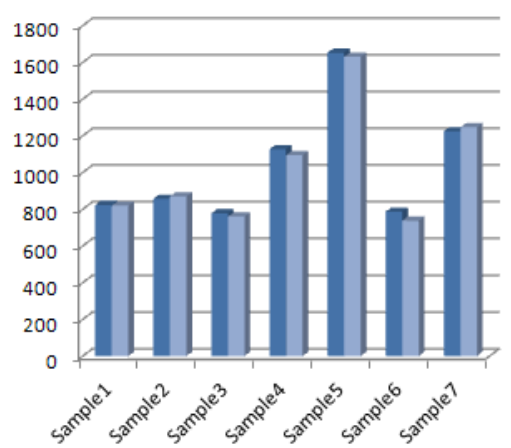

Calibrated Digital Numbers

$\left[\mathrm{Wm}^{-2} \mathrm{sr}^{-1} \mu \mathrm{m}^{-2}\right]$

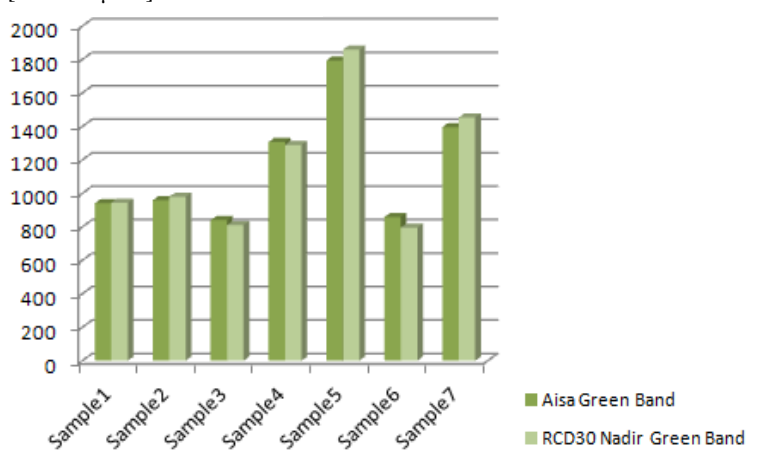

Calibrated Digital Numbers

$\left[\mathrm{Wm}^{-2} \mathrm{sr}^{-1} \mu \mathrm{m}^{-2}\right]$

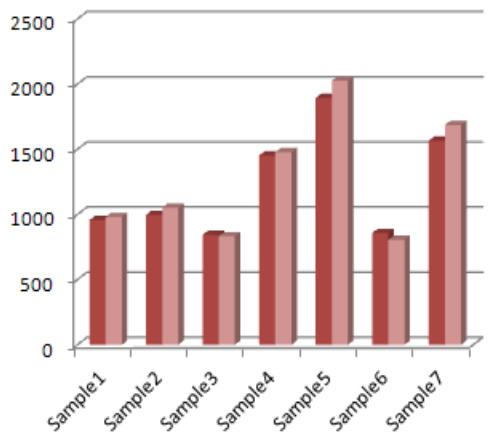

- Aisa Blue Band

InCD30 Nadir Blue Band

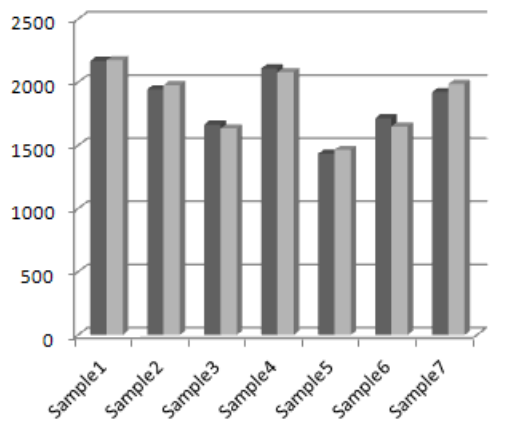

aisa NIR Band

nCD30 Nadir NIR Band
Figures 7,8,9,10. Histogram mean values computed for each sample and each band. Y axis represents Radiance

As a final summary of the previous measures, Table 1 shows the relative error of the RCD30 absolute calibration.

\begin{tabular}{|c|l|l|l|l|}
\hline & Blue & Green & Red & $\begin{array}{l}\text { Near } \\
\text { Infrared }\end{array}$ \\
\hline $\begin{array}{c}\text { Relative } \\
\text { error (\%) }\end{array}$ & 2.44 & 3.26 & 4.57 & 2.1 \\
\hline
\end{tabular}

Table 1. Relative error of the RCD30 absolute calibration

\section{IMAGE RESOLUTION}

Spatial resolution has become a key quality factor of the photogrammetric sensors, especially with the advent of digital sensors in the market. In this case, the spatial resolution is limited by the measure in pixel, yet it rarely coincides with the nominal resolution due to blurring and noise effects. They are caused by many factors. The main ones are the atmosphere (e.g. visibility, haze), the illumination geometry (sun height), the camera design (e.g. CCD, motion compensation, optic), the flight configuration and acquisition settings (e.g. flight speed, flight altitude, exposure, aperture) and data post processing (Read \& Graham, 2002).

The visual identification of targets was the most used procedure to assess the resolving power of an optical system. USAF 1951 was especially recognized amongst the rest of patterns due to it wider use. In the last decades, photogrammetric systems have shown important improvements of resolution capabilities, strengthening the importance of image quality. With the same aim, automatic methods developed to assess the performance of a system based on profiles of sinusoidal intensity and their evaluation by means of the Spread Functions (SF, image space) or the Modular Transfer Function (MTF, frequency space).

Since the acquisition of the first Z/I DMC camera, the ICGC has an in-house methodology supported by the Line Spread Function (LSF) intended to provide a resolving measurement of an image in pixel magnitude. The program processes a region of interest that contains a single contour and performs a minimum least squares adjustment over a bi-dimensional function of the edge modeled by a sigmoid function: 
The five parameters estimated by the least squares adjustment are used to achieve the analytical expression of the Line Spread Function in the image space. The FWHM (Full Width at Half Maximum) over the LSF is considered as the measurement value of the effective resolution.

\subsection{Theoretical expectations}

Image formation is performed by means of a Bayer filter demosaicking procedure for each camera head. This commonlyused color device typically alternates red and green filters for odd rows and blue and green filters for even rows: within each two-by-two cell, the green filters double the blue and red ones (Figure 11). Since demosaicking is carried out through a spatial interpolation approach, the final image gives better resolution values for green band in both Nadir and oblique images.

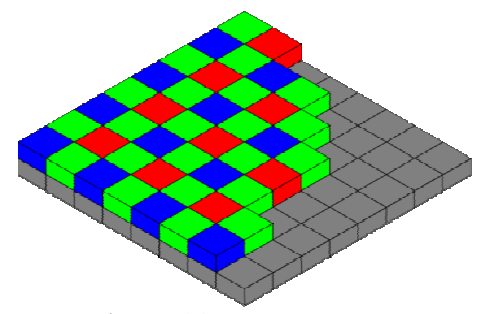

Figure 11. Bayer pattern

Furthermore, the resolution is not constant for oblique images since the image scale decreases with the increasing distance from the image center. The pixel size in the oblique image border on the ground $(\mathrm{x})$ is obtained from the geometrical relationships shown in Figure 12 and described in equations (3):

$$
\begin{aligned}
\mathrm{x} & =\mathrm{x}_{2}-\mathrm{x}_{1}, \mathrm{x}_{1}=\mathrm{h} \cdot \tan \left(\alpha+\alpha_{1}\right), \mathrm{x}_{2}=\mathrm{h} \cdot \tan \left(\alpha+\alpha_{2}\right), \\
\mathrm{d}_{2}=\mathrm{d}_{1}+\mathrm{p} & \\
\alpha_{1} & =\tan ^{-1}\left(\frac{d_{1}}{f}\right) \\
\alpha_{2} & =\tan ^{-1}\left(\frac{d_{2}}{f}\right)
\end{aligned}
$$

where:

$\alpha=$ tilt angle

$\mathrm{h}=$ flying height

$\mathrm{p}=$ pixel size in image

$\mathrm{f}=$ focal length

$\mathrm{d}=$ image side length $/ 2$

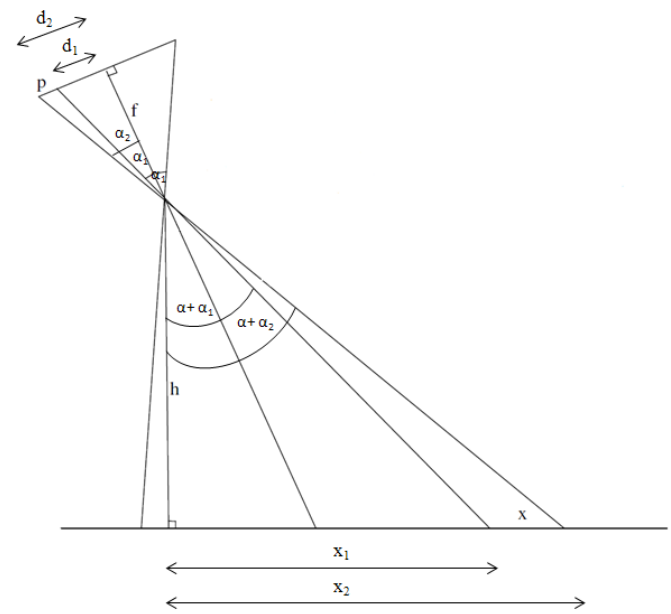

Figure 12. Geometry of a tilted camera

The scale reduction factors in the area of one component image (y direction) are shown in Figure 13. The graphic has been obtained by projecting a regular grid from object to image and comparing the distances of the points to nominal distances given by the nominal scale (Honkavaara et al., 2006). According to these reduction factors and the $5.2 \mu \mathrm{m}$ of pixel size, the resolution varies from a nominal RP value of 129 lines/mm to 74 lines/mm along the flight direction.

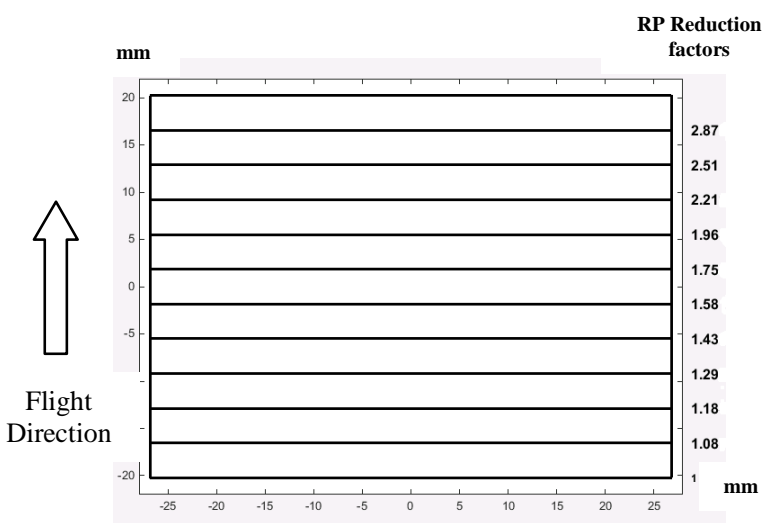

Figure 13. Resolution reduction factors for the Forward camera along flight direction. Position within the image is given in $\mathrm{mm}$.

\subsection{Results obtained with RCD30 aerial images and Siemens Star targets}

The algorithm has been applied on a Siemens star target painted on a canvas surface of $50 \mathrm{~m}^{2}$ (Figure 14) that has been captured for both Nadir and oblique cameras. Without loss of generalization, the analysis has been restricted to one oblique camera (Forward) which has acquired the bar pattern in 3 different images. In each of them the pattern was imaged at a different location within the image (T3 block-configuration). Moreover, in order to compensate for the directional effect of the measure, the algorithm has been applied on each edge of the pattern. The final effective resolution measure shown in Tables 2 and 3 has been calculated by averaging these values. 


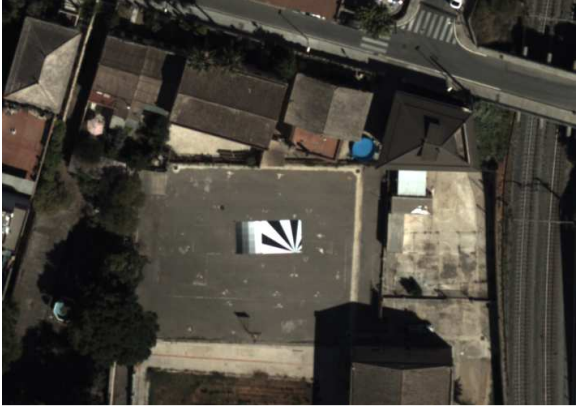

Figure 14. Siemens star quarter used for the resolution study

\begin{tabular}{|c|c|c|c|}
\hline $\begin{array}{c}\text { Resolution } \\
\text { (pix) }\end{array}$ & Red & Green & Blue \\
\hline Nadir & 1.9 & 1.3 & 2.2 \\
\hline Oblique & 3.1 & 2.4 & 3.6 \\
\hline
\end{tabular}

Table 2. Effective resolution results for Nadir images in pixels

\begin{tabular}{|c|c|c|}
\hline Forward & $\begin{array}{c}\text { Pattern location } \\
\text { in image (y axis, } \\
\text { in milimeters) }\end{array}$ & $\begin{array}{c}\text { Resolution } \\
\text { (pixel, green } \\
\text { channel) }\end{array}$ \\
\hline Image 1 & -9.38 & 2.23 \\
\hline Image 2 & 6.16 & 2.41 \\
\hline Image 3 & 18.31 & 2.60 \\
\hline
\end{tabular}

Table 3. Resolution measurements as a function of the location in the image

These results prove that the image formation procedure has the major influence on the effective resolution. Typically, cameras with a single CCD for every channel, such as DMC, show a worsening of the effective resolution as the wavelength decreases due to Rayleigh scattering. Conversely, RCD30 presents the best resolution values in green channel, while the gap between red and blue values appears as a consequence of atmospheric dispersion. In addition, mainly for the oblique cameras, the values show that the location of the object within the image greatly affects the resolution, since the tilted geometry leads to different optical path lengths for every pixel. Finally, the reduction factors obtained for oblique images must be taken into account when translating the pixel value into the object space. As a consequence, the ground sampling distance will not be constant.

\section{STEREO PLOTTING}

Besides the geometric and radiometric assessments, a qualitative analysis on the usability of the RCD30 Oblique imagery for stereo plotting purposes has been carried out. Stereo pairs have been generated using Nadir images and visualized in a $3 \mathrm{D}$ environment in order to evaluate the usefulness of these images in a production workflow for creation and updating of topographic databases covering urban areas with a level of detail equivalent to 1:1000 scale (T1 block-configuration). The approximately $70 \%$ overlap is not the best configuration to achieve a good Base-Height ratio, but the use of only alternative images for the generation of the stereo pairs was discarded because they would not cover completely the area.
In the stereo plotting tests three aspects have been considered: the visualization of the stereo pairs, the photointerpretation of the real world objects and the positional accuracy of the digitation.

Related to the quality of the images, it has been seen that the main limitation of RCD30 Oblique images is the high number of fuzzy images, which hinder the stereoscopic vision and visual fatigue, and may provide poor accuracy in positioning and measuring. However, since the production phase is still in an early state of deployment, the reasons concerning blurred images have to be analyzed in more detail.

Figure 15 shows an example to illustrate the reason of the stereoscopic problems.

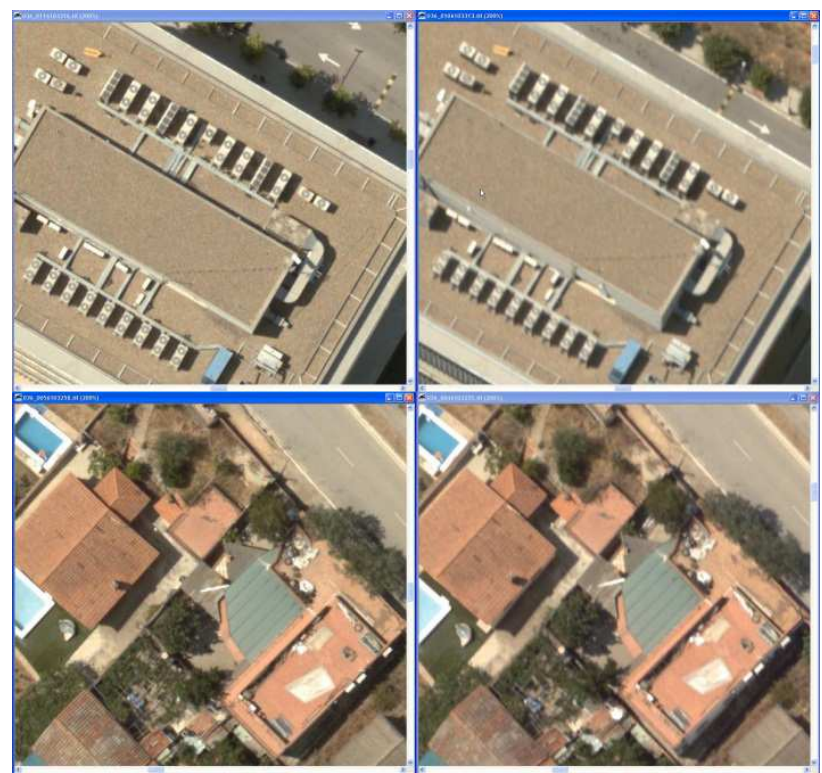

Figure 15. Clearness differences between left image and right image of the same stereoscopic model.

Another problem detected is the loss of resolution in the extremes of the images (section 5), as is showed in the road of Figure 16, which also makes uncomfortable data capture and updating.

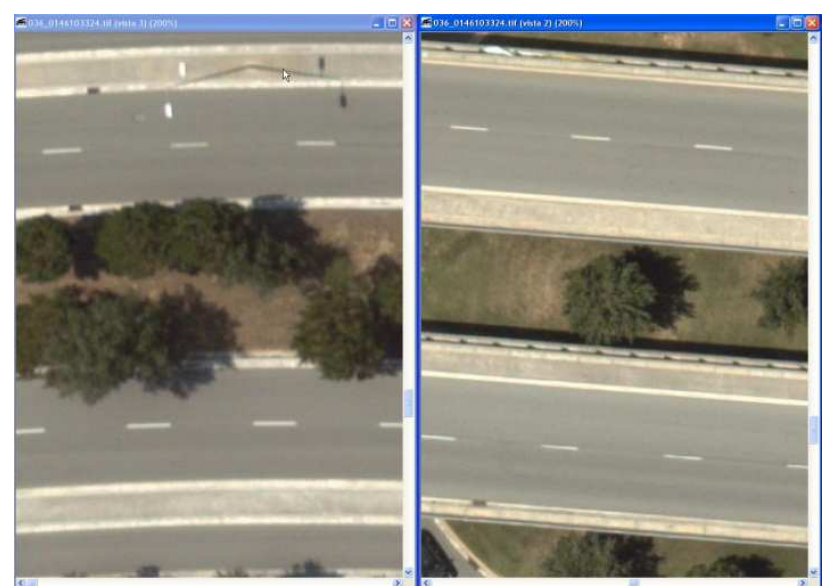

Figure 16. Clearness differences between the extreme part (left) and centre (right) of the same image. 
The photointerpretation has been based on the ICGC catalogue that includes the features and the attributes collected in the urban topographic database at 1:1000 scale. Using the RCD30 stereomodels, the recognition of the required level of detail for this database has widely achieved.

As in the case of aerial triangulation, the positional accuracy of stereoplotting has been assessed by comparing the coordinates of independent check points with the coordinates of the same point measured in the stereoplotter. Table 4 allows comparing the positional accuracy achieved with the RCD30 images and the DMC aerial images at $9 \mathrm{~cm}$ nominal pixel, in which usually is based the ICGC stereoplotting process. The positional accuracy required for the topographic database at scale 1:1000 is $20 \mathrm{~cm}$ in $\mathrm{X}, \mathrm{Y}$ and $25 \mathrm{~cm}$ in $\mathrm{H}$.

\begin{tabular}{|l|c|c|c|}
\hline & $90 \% \mathrm{X}(\mathrm{m})$ & $90 \% \mathrm{Y}(\mathrm{m})$ & $90 \% \mathrm{H}(\mathrm{m})$ \\
\hline $\begin{array}{l}\text { RCD30 Nadir } \\
\text { images }\end{array}$ & 0.08 & 0.07 & 0.10 \\
\hline DMC images & 0.143 & 0.150 & 0.169 \\
\hline
\end{tabular}

Table 4. Positional accuracy.

An additional positional check has been performed comparing the results of measuring a point from different stereo pairs. This comparison provides an estimate of the different cumulative errors from the orientation and positioning processes. Table 5 shows that the differences in measurements from different stereo pairs are, in comparison with to the DMC images, better in coordinates $\mathrm{X}$ and $\mathrm{Y}$ and not so good in elevation.

\begin{tabular}{|l|c|c|c|}
\hline & $\mathrm{X}(\mathrm{m})$ & $\mathrm{Y}(\mathrm{m})$ & $\mathrm{H}(\mathrm{m})$ \\
\hline $\begin{array}{l}\text { RCD30 Nadir } \\
\text { images }\end{array}$ & 0.05 & 0.05 & 0.16 \\
\hline DMC images & 0.09 & 0.08 & 0.11 \\
\hline
\end{tabular}

Table 5. Comparison between RCD30 and DMC measurements resulting from orientation and positioning

This loss of accuracy in elevation, compared to the good results obtained in the coordinates $\mathrm{X}$ and $\mathrm{Y}$, are likely due to the poor Base-Height ratio of the stereo pairs mentioned above.

\section{SUMMARY AND CONCLUSIONS}

RCD30 Oblique images prove to be suitable for all the purposes analyzed in the study. Preliminary results in aerial triangulation have shown that sub-pixel accuracy can be routinely achieved in production flights. Nevertheless, efficient time consumption for image matching and least-squares bundle adjustment involving the five cameras is a key point to improve in order to achieve a efficient production line. Taking advantage of the redundant information generated through the five cameras and the high overlaps, preliminary dense point clouds and subsequent textured meshes have been generated using the Bentley Smart3DContextCapture software package and T1 blockconfiguration.

Concerning the radiometric calibration a validation process of the workflow included in the post-processing software package has been proposed. The methodology developed -based on the comparison between AISA Eagle II and RCD30 simultaneous acquisitions- has demonstrated the suitability of the RCD30 calibrated data for remote sensing applications.
Regarding the resolution of RCD30 images resolution measures have been generated from a Siemens Star target whose dimensions are known. The measurement FWHM extracted from the LSF function provides a specific quantification of the resolving power of the system. The results have shown the impact of the image formation process (Bayer filter) on the effective resolution, as well as the position of the objects within the image due to the different optical path length that has to be covered for every pixel. In addition, the description of the tilted geometry for the oblique cameras has been included in the section. The projection of a regular grid from the object to image space and the comparison between distances of the points to nominal distances have provided the reduction factors of the resolution along the camera pointing direction.

From the stereoplotting point of view, the conclusion is that RCD30 stereopairs from Nadir images are suitable to achieve the positional accuracy and to identify and classify properly the elements included in the catalogue of the ICGC topographic database at 1:1.000 scale, which are the required to create or update the information on urban areas. Main limitation using the RCD30 images is related to the resolution variation within the images, which difficult the stereoscopic vision and produce visual fatigue. Preliminary results for positional accuracy have allowed us to provide firsts comparison values between DMC and RCD30. However, future tests at different scales and different configuration overlaps must be carried out to generalize the comparison in a more rigorous manner.

\section{ACKNOWLEDGEMENTS}

We would like to thank the hard job done by Andrés Alvarruiz and Joan Marimón for the measurements of the check points used in aerial triangulation and stereoplotting assessment. Furthermore, we really appreciate the collaboration of Joan Arnaldich in the development of specific software tools to efficiently handle the increase of data involved, as well as the cooperation of Luca Pipia, Anna Tardà and Ramon Alamús with the AISA Eagle II flight survey planning and data processing.

\section{REFERENCES}

Ebner, H., 1976.Self-calibrating block adjustment, ISP-congress Helsinki 1976, Int. Archive of the ISPRS, Vol 18-3

Grün, A., 1979. Self-Calibration versus Testfield Calibration, ISP WG III/3, Aalborg 1979.

Read, R.E, Graham, R.W.,(2002).Manual of Air Survey: Primary Data Acquisition. Whittles Publishing, Caithness. $408 \mathrm{p}$.

Honkavaara, E., Jaakkola, J., Markelin, L., Becker, S., 2006. Evaluation of Resolving Power and MTF of DMC. In proceedings of ISPRS Comission I Symposium, Paris. 